\title{
An Optimal Approach on Sensor Placement for Structural Vibration Survey
}

\author{
Chunhui Yuan ${ }^{1, *}$, Weicai Peng ${ }^{1}$ and Junjie Zhang ${ }^{2}$ \\ ${ }^{1}$ China Ship Development and Design Center, Wuhan 430064, China \\ ${ }^{2}$ National Key Laboratory on Ship Vibration \& Noise, Wuhan 430064, China \\ ${ }^{*}$ Corresponding author
}

\begin{abstract}
Vibration test on hull structures with large size needs numerous amounts of sensors, which means too much work. However, notable error would come from reducing the number of sensors. An approach is proposed to reconstruct the complete structural vibration, which chooses the locations and the number of sensors optimally based on the condition number of structural modal matrix. This iterative progress eliminates the sensor location that contributes the most significantly to the condition number of modal matrix in each cycle. The influence of the location number eliminated in each cycle is analyzed and emulated. An experiment validates the precise of this approach; the error is only 3.0 dB. The distributions over the surface of structure are also consistent between reconstructive and measuring data.
\end{abstract}

Keywords-vibration; survey; sensor placement; condition number

\section{INTRODUCTION}

Hull structure is the main source of radiated noise. Thus it is an important way to inspect stealth condition by vibration survey. However, Vibration measurement on large-sized structures is difficult as far as the uphill work is concerned. Hundreds of sensors have to be placed on the structure by hands, together with long signal cables. This means not only hard work, added mass to the structure may influence its dynamic characteristic. On the other hand, survey by insufficient sensors is risky with respect to data discrepancies. Thus optimal sensor placement for vibration survey on large structure is necessary. One can reconstruct the complete vibration response field of the structure using limited data measuring by these chosen sensors. This method can substitute the way that measuring every inch over the surface, which gets sufficient spatial resolution of response information about the structure.

Lots of methods of optimal sensor placement have been proposed. One common measure to judge the suitability of sensor positions is the Fisher information matrix (FIM) using mode shapes of the structure. Kammer proposed this Effective Independence (EFI) method to quantify the contributions of response measurements so that the modal states of targeted modes can be optimally observed [1]. Similar studies of FIM are proposed to choose sensor locations by maximizing the determinant of the FIM, by maximizing the smallest eigenvalue of the FIM, by minimizing the trace of inverse of the FIM, by maximizing the norm of the FIM, or by minimizing the condition number of the FIM. Another criterion to judge combinations of sensor positions within single setup configurations is the modal kinetic energy. Heo et al. derived the Kinetic Energy Optimization Technique (EOT) with the formulation similar to EFI [2], and the difference lies in the quantity that is optimized. The EFI method maximizes the Fisher' $s$ information matrix while the EOT optimizes the kinetic energy matrix. As the kinetic energy is only a mass weighted version of the Fisher information matrix, the connection to the effective independence method is obvious. However, EOT eliminates the problem of EFI that the sensor locations with low energy content may be selected. A similar way to solve the problem is the EFI-DPR (Driving Point Residue) method, which multiplying the candidate sensor contribution of the EFI method with the corresponding DPR coefficient [3]. Another criteria is derived from the modal assurance criterion (MAC) originally introduced by Allemang [4], whereas the off diagonal terms of the MAC matrix need to be minimized.

Condition number is also been used for condense sensor group, which is well known as a measure of robustness of the system. The criterion can be the condition number of the observability matrix of the linearized tangent model of the discretized model of the process [5], the spectral condition number of the Hankel matrix [6], or the condition number of the frequency response function [7]. In this article, the condition number of the modal matrix of structure is employed to optimize sensor locations. Experiment demonstrates the accuracy of this approach and its results are illustrated later.

\section{APPROACH OF CONDITION NUMBER}

Vibration responses of the structure can be expressed in mode shapes:

$$
V=\phi A
$$

where $V$ is the vector of responses, $\phi$ is the matrix of mode shapes, $A$ is the vector of modal coordinates.

In fact, not all degrees of freedom can be given in mode shapes or placed sensors. In sensor location optimization, a limited number of sensors are placed on the chosen measurable degrees of freedom. Therefore, the chosen DOFs are given in the formulation, and secondary ones are omitted. This is the purpose of optimization that condensing enormous sensor candidates into much smaller group so that the latter can be applied to engineering practice. 
If only the primary DOFs are employed in Eq. (1), it can be rewritten as:

$$
V_{E}=\phi_{E} A
$$

where $V_{E}=\left[v_{1}, v_{2}, \ldots, v_{E}\right]^{T}$ represents the chosen velocity responses of $\mathrm{E}$ locations, and $\phi_{E}$ is the corresponding matrix of mode shapes at the chosen locations.

According to Eq. (2), there is:

$$
A=\phi_{E}^{+} V_{E}
$$

where ' + ' denotes the Moore-Penrose generalized inverse matrix.

Substituting Eq. (3) into Eq. (1), one gets

$$
V=\phi\left(\phi_{E}^{+} V_{E}\right)
$$

Eq. (4) means that one can measure only few responses and reconstructs responses anywhere over the structure (suppose all mode shapes are available). However, these few sensor locations are not chosen freely. The combination of sensor positions dominates the accuracy of inverse problem as well as the accuracy of response reconstruction. Herein the approach of condition number is employed to choose the combination of sensor locations for optimization.

The condition number of $\phi_{E}$ dominates the accuracy of the matrix inverse in Eq. (3). The bigger the condition number of $\phi_{E}$ the bigger the error is. So one can chooses the group of sensor locations leading to the smallest condition number of $\phi_{E}$, then the optimization of sensor placement comes to realization. Herein the condition number of $\phi_{E}$ is the criteria of optimal sensor placement, it can be expressed in Frobenius norm:

$$
\operatorname{cond}\left(\phi_{E}\right)=\left\|\phi_{E}\right\|_{F}\left\|\phi_{E}^{+}\right\|_{F}
$$

where \|\|$_{F}$ denotes Frobenius norm.

The number of optimal locations E should be bigger than the number of vibration modes participated in the calculation. In practice more sensors should be placed for measurement as various uncertainties are concerned. In the approach of condition number proposed in this article, one can get this final optimal number of location according to the rising speed of condition number during the iteration.

\section{DIFFERENCE BETWEEN ITERATION AND ACCOMPLISHING OPTIMIZATION ONLY ONCE}

According to the approach mentioned above, reducing mode matrix by its condition number can achieve choosing survey locations of number $\mathrm{n}$ from primary locations of number $\mathrm{m}$. The rational procedure is accomplishing it only once, which means reducing locations of number $m-n$ simultaneously. However, this needs inverse matrix calculations of number $C_{m}^{n}=\frac{m !}{n !(m-n) !}$ with tremendous consumption. A substitute is iteration which reduces only one location in an iteration cycle. This needs cycles of number $(m-1)+(m-2)+\ldots+(m-n)=\frac{n \times(2 m-n-1)}{2}$ which far less than the former. For example, it will costs $C_{200}^{50}=\frac{200 !}{50 ! 150 !}=4.5386 \times 10^{47}$ matrix inversion cycles choosing 50 locations from 200 for the former, and only $\frac{n \times(2 m-n-1)}{2}=8725$ iteration cycles for the latter.

The question is if the result locations of these two processes are the same or equivalent. Theoretically speaking, the approach of condition number reduces symmetric locations of the structure that contain similar mode information. As a result the left choosing locations are asymmetric. On the other hand, locations containing different mode information are reserved because the more modes participate the less similar vibrations appear. Accomplishing optimization only once or choosing locations by iteration all reserve locations of complete independence, thus they are equivalent in location optimization.

To prove the equivalence, an ANSYS model was adopted to choosing 198 locations from primary 200 location candidates by the approach of condition number. The result shown in Fig. I exposes that reduced locations by two different processes are symmetric on the structure. These symmetric locations contain the same mode information. They have similar influences to the condition number of mode matrix, so it is equivalent to reduce any one of symmetric locations.

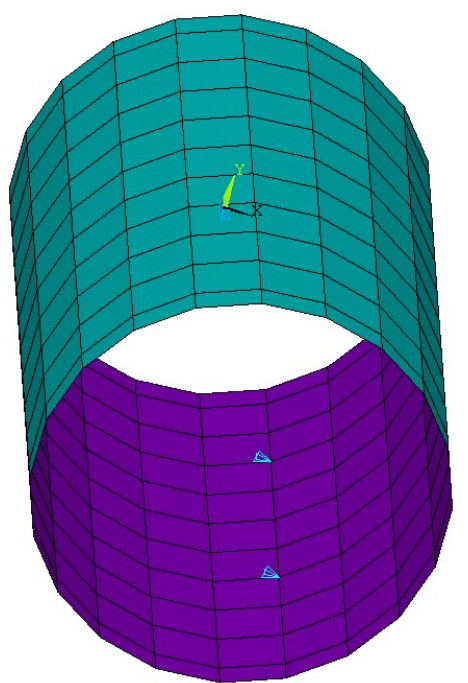

(a) 


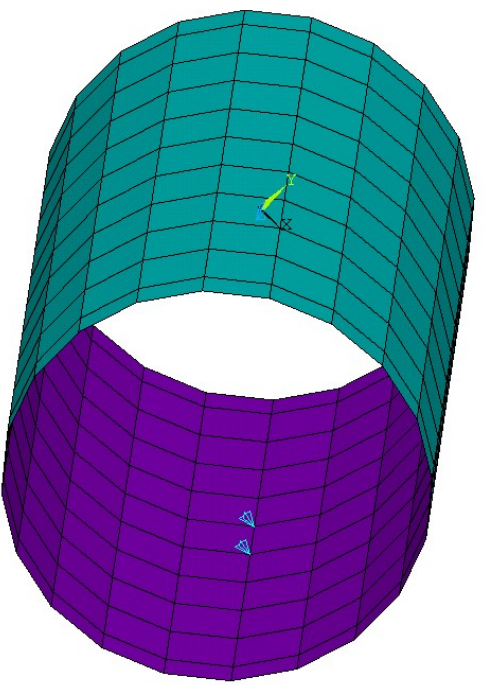

(b)

FIGURE I. TWO EXAMPLES OF REDUCED LOCATIONS(TRIANGLES INDICATE REDUCED LOCATIONS BY TWO DIFFERENT PROCESSES)

\section{EXPERIMENT AND RESULTS}

\section{A. Experimental Set-up}

In order to demonstrate the approach of condition number mentioned above, an experiment is carried out on a cylindrical shell structure. 264 sensors are placed uniformly over the inner surface of the cylindrical shell. Responses of the structure are excited by an electromagnetic vibration generator isolated from the shell by isolators. The cylindrical shell is sealed and sunk into the water.

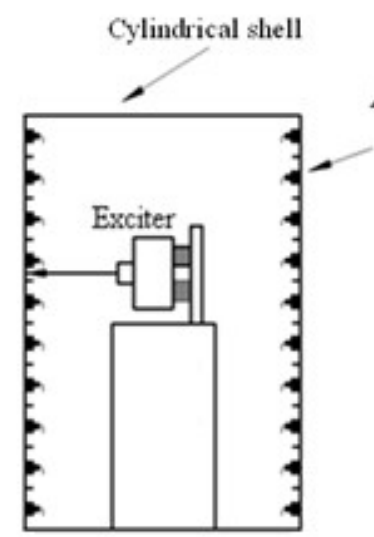

Acceleration sensor

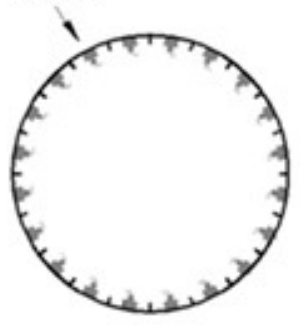

Elevation

Planform

FIGURE II. SKETCH OF EXPERIMENTAL STRUCTURE AND SENSOR PLACEMENT

The aim of the experiment is that measuring vibration responses of the cylindrical shell by limited sensors at optimal locations, and then reconstructing the complete responses of the structure. The reconstructive results are compared with the real data measuring by 264 sensors in uniform placement to

verify the consistencies. The highest frequency of concern is $100 \mathrm{~Hz}$.

\section{B. Experimental Results}

During iteration, the condition number goes down quickly to about 10 , and then keeps steady approximately until rising suddenly after deep iterations. Since the more iteration cycles the fewer the optimal sensor locations left, one should accept results after the more cycles the better. Meanwhile a smaller condition number should be guaranteed. Thus one can choose to stop iteration before the condition number zooming shown in Fig. III, and the corresponding number and locations of the left DOFs (where to place sensors finally in practice) are optimal. In Fig. III we choose 27 final sensor locations to reconstruct the complete structural vibration, and the corresponding condition number of submatrix of mode shapes is about 10 .

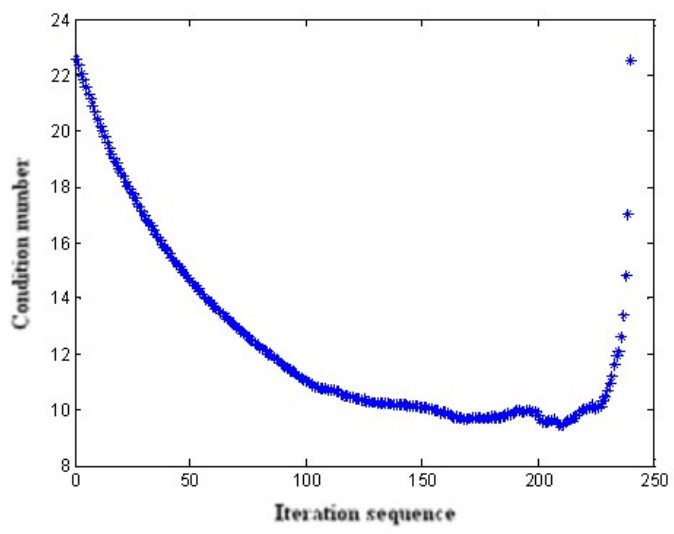

FIGURE III. CONDITION NUMBERS DURING ITERATION

Vibration responses of the structure are reconstructed by Eq. (4) using the measured data of the optimal 27 sensor locations. The error is only $3.0 \mathrm{~dB}$ according to vibration levels of RMS in the frequency band lower than $100 \mathrm{~Hz}$, averaged over the surface of the structure. The response distributions on the surface of the cylindrical shell are compared between reconstructed and measured results. Typical results shown in Fig. IV demonstrate good consistencies of response distribution.

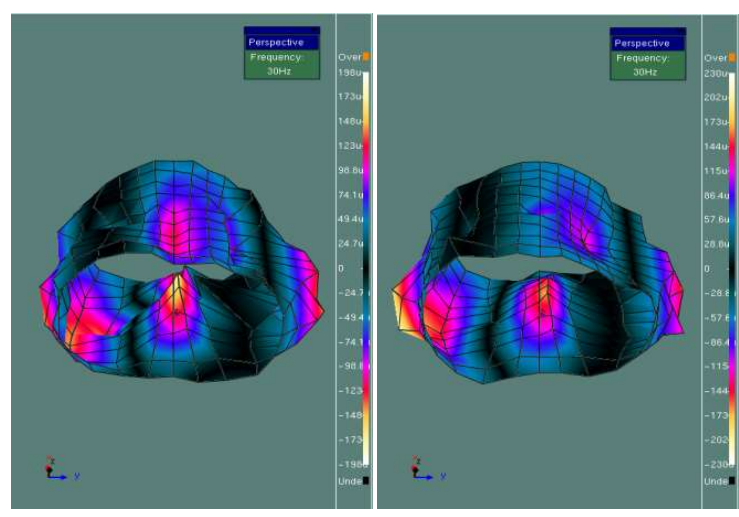




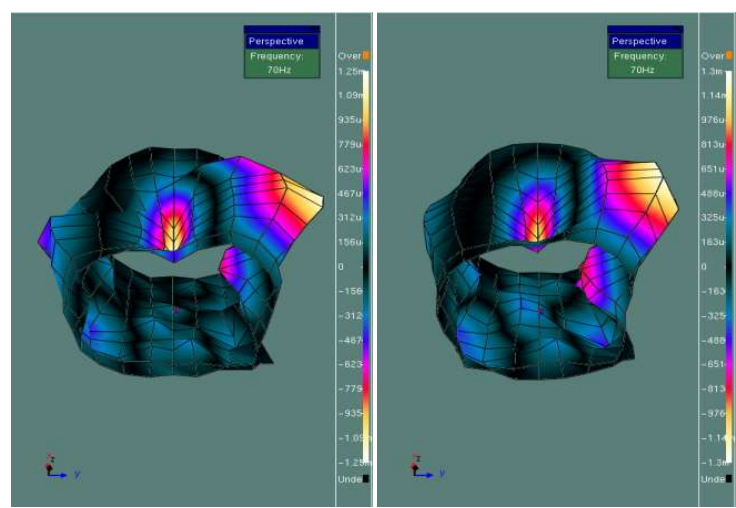

FIGURE IV. TYPICAL COMPARISON BETWEEN RECONSTRUCTED (LEFT) AND MEASURED (RIGHT) RESPONSE DISTRIBUTION

\section{CONCLUSiOnS}

The approach of condition number for optimal sensor placement proposed in this article is effective and validated by the experiment, which reconstructs the complete structural vibration via much fewer sensors. It reduces sensor locations that contribute significantly to the condition number of modal matrix in iteration. The reconstructive error is only $3.0 \mathrm{~dB}$ comparing 27 optimal sensor locations with 264 initial sensor placements. The distributions over the surface of structure are also consistent between reconstructive and measuring data. This approach may be applied to vibration survey on large structures.

\section{REFERENCES}

[1] Kammer DC. Sensor placement for on-orbit modal identification and correlation of large space structures. Journal of Guidance, Control, and Dynamics. 1991, 14 (2), p.251-259.

[2] Heo G, Wang ML, Satpathi D. Optimal transducer placement for health monitoring of long span bridge. Soil Dynamics and Earthquake Engineering. 1997, 16 (7-8), p.495-502.

[3] Meo M, Zumpano G. On the optimal sensor placement techniques for a bridge structure. Engineering Structures. 2005, 27 (10), p.1488-1497.

[4] Allemang RJ, Brown DL. A correlation coefficient for modal vector analysis. Proceedings of the 1st International Modal Analysis Conference, Orlando, Florida 1982, p.110-116.

[5] Dochain D, Tali-Maamar N, Babary JP. On modelling, monitoring and control of fixed bed bioreactors. Computers Chem. Engng. 1997, 21(11), p.1222-1266.

[6] Li YY, Yam LH. Sensitivity analyses of sensor locations for vibration control and damage detection of thin-plate systems. Journal of Sound and Vibration. 2001, 240(4), p.623-636.

[7] Choi HG, Thite AN, Thompson DJ. Methods for selecting sensor locations for improving indirect force determination. Proceedings of the Institute of Acoustics. 2004, 26(2), p.265-276. 